\section{Academic researchers' use of the Internet, and their consequent support requirements}

\author{
Neil Jacobs
}

\section{Reasons for the research}

The internet cannot be ignored by library professionals. Indeed librarians have been key players in the development of useful WWW resources, and in supporting those who would like to use those resources. The novel features of the internet as an information medium suggest, however, that its use may not be best supported by traditional library approaches. It was for this reason that the University of Sussex Library proposed to investigate how local academic researchers were using the Internet in their work (if at all), and thereby to assess their support requirements. In 1995 the University of Sussex Research Development Fund accepted a proposal for the Library to undertake a project, one of whose key objectives was to assess the practical value to research at the University of Sussex of internet resources, and the consequential training and support needs.

Many authors have noted that the contexts in which new information technologies are used both shape that use, and are themselves shaped by it. Barry ${ }^{(1)}$ has put it as follows:
"The electronic library and the Internet are altering the nature of research in academia. Information behaviour in research is changing: information seeking, information retrieval, information management and communication of information are all being affected by the move from traditional to Information Technology (IT)-assisted information methods."

It was for this reason that we thought it timely to investigate some aspects of researchers' information behaviour. Furthermore it seemed at the start of the project (and still seems at the time of writing) that the World Wide Web (WWW) format is acting as a point of convergence for several sources of information relevant to researchers. These sources include journals, preprint archives, and publications from nongovernmental and governmental organisations around the world. The convergence of these sources onto the WWW led us to believe that the library should be aware how this important medium was being used by academic researchers, if at all. Locally, the University of Sussex Library had developed a number of support and training 
structures for University members who wanted to use the internet. There were training sessions, workshops, helpsheets, and a local internet gateway structure, the Pier ${ }^{(2)}$. We felt that these efforts needed assessing, and that the time was right to undertake a review of the likely future directions of the support requirements of our users in this area.

\section{An account of the research}

To look at the information worlds of a wide variety of researchers across an entire university was a larger project than there were funds to undertake. We had to limit the scope of the work somehow, and we decided to focus on those subject areas where we thought that the internet might have greater potential. These were largely social science subjects where there were frequent requirements for access to up-to-date, nongovernmental and foreign-sourced material.

From the background to the project, it is clear that we wanted to ask 'how' and 'why' questions:

- 'how is the Internet being used in conjunction with other resources?'

- 'why is this academic not using it at all?'

At this stage we were less interested in 'how many' questions:

- 'how many accesses do Sussex researchers make to a particular site?'

The appropriate methods for our work were therefore qualitative.

We interviewed 30 researchers during the first of two rounds of interviews, and 6 during a second round. The latter figure is so low that information from the second round of interviews has been largely discounted. The first round of interviews had three main aims: to establish the researchers' technical expertise and setup; to explore their use of Internet-based resources; and to discover the main advantages and problems of using those resources. The interviews were semi-structured, so that while a checklist of topics was used to ensure that participants gave their views on key themes, there was also scope for them to emphasise other aspects of their work that they felt were relevant. Extensive notes were taken during the interviews, and were written up immediately afterwards. We entered these notes into the qualitative data analysis software package NUD*IST ${ }^{(3)}$ and coded them according to a set of themes relevant to the project's aims and to the researchers' comments. This enabled us to pull out from our notes the issues that seemed to really matter, and to group them together to present the Library and University management with a clear and structured report of the project's findings. We divided our findings and recommendations into four: technical issues; support issues; training . issues; and the local Internet gateway.

\section{Technical issues}

It was not the purpose of the project to carry out a thorough review of the suitability of researchers' computers and network connections. However we did gain some impression of this important aspect of the context in which researchers use the Internet. We found that there were wide variations in the hardware, software and network configurations of researchers, and that these configurations depended largely on initiatives from individuals or academic units. In particular it was clear from researchers' comments that some of the arrangements of the local area network were seen by some as needlessly frustrating, that the campus network appeared at times to be prohibitively slow, that 386 machines were inadequate for accessing the WWW, and that some researchers wanted to access the WWW from home via the University Computing Service. These perceived problems were technical (or financial) at source, but researchers talked about them in terms of their need to economise on time.

\section{Support issues}

The major recommendations of the research project were that the role of information gatekeeper should be established in each discipline, and that much day-to-day computer network support should be devolved to academic units. These conclusions were in line with those of another recent University report, which recommended that:

\footnotetext{
"each unit identify a member of staff responsible for computing support and that role be specified in their job specificationequally, each discipline (etc.) should prepare to name someone responsible for the identification, testing and reporting on discipline-specific software." (4)
} 
We recommended that the discipline-based role be extended to include the establishment and maintenance of a subject-specific internet gateway (see below). Since researchers expressed a demand that support for the use of subjectspecific information resources be available close at hand and at the moment of need, we recommended that the discipline-based role should include responsibility for providing a first line support service for researchers' use of networked information sources. The role of coordinating the new decentralised staffing structure would fall to the University of Sussex Library and Computing Services, and we recommended that these two units begin discussions on the best ways to achieve such coordination

\section{Training issues}

The project could not serve as a full evaluation of the training initiatives thus far undertaken, since not enough researchers were interviewed. We found that no researcher was using Internet-based resources to their full potential, and all were missing some knowledge or skill that could improve the effectiveness of their Internet use. However we also found that many researchers felt that they ought to have these information skills already, being practising professionals in their field: they expressed strong reservations about volunteering for training sessions. The project therefore recommended that future training initiatives should emphasise that new skills are required to make use of Internet-based resources, and that, despite the apparent ease of use of the WWW, self tuition is not the best way to acquire these skills. Furthermore, because researchers are concerned to economise on time, they want training that is specific to their information needs. Academics wanted tuition and help that was close at hand, relevant to their particular technical setup, and available at the moment of need. We therefore recommended that the overall training and support effort of the University of Sussex Library and Computing Service be appropriately divided between those initiatives that could best be undertaken by professional central support staff, and those that were more effectively delivered by the discipline-based and/or unitbased information gatekeepers mentioned above.

\section{The local Internet gateway - the Pier}

Those researchers who used the Pier expressed enthusiasm for (though limited knowledge of) its structure. Those who used other methods for locating and accessing Internet-based resources expressed some uncertainty as to the role of a local gateway. We found that there were useful roles that could be played by a local Internet gateway, but that these roles should be made explicit. They were to act as:

- an intermediary stage between personal bookmark files and the major indexes / search tools;

- a clearing house and accessible repository for links that individuals within a discipline or subdiscipline feel would be useful to local colleagues;

- a starting point for novice users of the Internet;

- a training aid.

The first two of these suggested that the local gateway could be marketed as a time-saver for researchers.

The fact that it was possible to identify such roles meant that we recommended that a local internet gateway structure be maintained and, where appropriate, enhanced. However, given that its roles are closer to the academic unit than a central library, we felt that this maintenance should be undertaken by the discipline-based information gatekeepers (as mentioned above), rather than centrally by Library staff. Furthermore we felt that they should ensure effective feedback and suggestions from the researchers who used the service. The discipline-based areas of the Pier may be hosted on servers around campus, but the requirements of support for interdisciplinarity meant that we recommended that the whole gateway should appear to the user as an integrated service. Some areas of the Pier are relevant across many or all disciplines. Hence we concluded that a core gateway could be developed from the current Pier structure, and be managed and maintained by central Library staff.

\section{Other relevant research}

The project at Sussex has complemented work done elsewhere. Of this work perhaps the most relevant has been that undertaken by Paul Hollands at Loughborough University, Christine 
Barry at the School of Education, King's College London, and the TAPin (Training and Awareness Programme in networks) eLib project.

Paul Hollands ${ }^{(5)}$ undertook a quantitative survey of academic researchers to establish their familiarity and use of a range of Internet services. However it is difficult to understand the percentages given in his report with no knowledge of the context in which the survey responses were given, and this is the drawback of much purely quantitative work. Christine Barry ${ }^{(6,7)}$ has written about a qualitative study that was concerned to understand the use and non-use of different aspects of the 'digital library'. Her work applied to many more electronic resources than just the Internet, so that we were unsure to what extent her findings were relevant to the Internet in particular. In fact her findings were remarkably similar to ours. In particular she found that:
"The increasing pressures on the academics in terms of increased administrative responsibilities, pressure to publish and increasing difficulty in gaining research funding. All of which mean they have less time for information-seeking and less time to learn to use the digital library." and "In the past academics have tended to teach themselves to use software and they try to apply this method to learning about the digital library, this can cause problems as the digital library systems are rarely self-evident and misuse can result"

Her conclusions were also similar to the recommendations or our project. Most pertinently she concluded that:

"Librarians will have to go out into the organisation. This suggests a shift from the traditional reactive role of the librarian to becoming integral players in the departments outside the library... The increased need for information skills training, required by the electronic information world, becomes too great a task for librarians to achieve alone... Academic staff are better placed in some ways, than librarians to link information skills to purposeful information-seeking.

Also, training in context, related to academic subject tasks, is preferable to abstract generic training."
All three of these quotations would be strongly endorsed by the findings of the TAPin project ${ }^{(8)}$ which looked at academics in several universities, and which came to very similar conclusions. Unfortunately the TAPin project was running concurrently with the work at Sussex, so that its findings were not available until our work was nearly done. One other project, which at the time of writing has yet to report, is BLERBS ${ }^{(9)}$ at the University of Kent at Canterbury. Their emerging concept of the 'barefoot librarian' may have similarities with the information gatekeepers recommended by our project.

\section{Conclusions}

It would seem that there is a consensus within the work done in this area, that for the Internet, support from the library to users should be close to the users. It should also be specific to the users' particular information world. Hence the move toward 'information gatekeeper' or 'barefoot librarians'. However, although our final report (with these recommendations) has been accepted for the most part by the Library at Sussex, a severe lack of funds within the University as a whole means that - at the time of writing - there is no chance of these support staff and structures coming into being. The implication must be that researchers at Sussex will not get the support they need to make best use of the rapidly changing 'digital library'.

\section{Notes and References}

1. Barry, C Training the next generation of academic researchers to operate in an electronic world. SIG ENET roundtable presentation: American Educational Research Association conference April 11th 1996. Also available on the WWW at: http://www.kcl.ac.uk/kis/schools/ education/cbaera.html

2. The Pier is at: http://www.sussex.ac.uk/library/pier/

3. For a description of the package and its use, see (for example): Jacobs, NA Students perceptions of the Library service at the University of Sussex: Practical quantitative and qualitative research in an academic library, Journal of Documentation 52(2) June 1996, 139-162.

4. Report of the Computing Service and 
Information Infrastructure Review Group, University of Sussex, 1996. para 16.34.

Readers should be aware that 'disciplines' and 'units' are cross-cutting structures at Sussex, due to the emphasis on interdisciplinarity.

5. Hollands, P Results of the Internet and Information sources and Services Survey. Available at: http://www.lboro.ac.uk/info/training/report/

6. Barry, C. Training the next generation of academic researchers to operate in an electronic world. SIG ENET roundtable presentation:: An American Educational Research Association conference April 11th 1996 Also available on the WWW at: http://www.kcl.ac.uk/kis/schools/education/ cbaera.html

7. Barry, C. The Digital Library: the needs of our users. paper presented at the International Summer School on the Digital Library, 5th August 1996, Tilburg University, The Netherlands. Also available on the WWW at: http://kubax6.kub.nl:2080/summer/96/ abstract/Barry.html.

and at http://www.kcl.ac.uk/kis/schools/education/ cbticer.html

8. Flatten, K, Mackenzie, $\mathrm{N}$ and Elkin, J TAPin: Training and Awareness Programme in networks. Interim Report, March 1996. The full annual report is now available from the University of Central England in Birmingham, and on the WWW at: http://www.uce.ac.uk/tapin/reports/annual.htm

9. British Library Ethnographic Research on Bibliographic Services, details on WWW at: http://lucy.ukc.ac.uk/Blerbs/ 